\title{
Der 'Pantokrator': Die Inszenierung von Gottes Macht in der Offenbarung des Johannes
}



God's power according to Revelation. The article argues that the 'power of God' expressed by the title 'Pantokrator' in the Revelation of John should be interpreted against a concrete context and issues about God's deeds of righteousness. From a human perspective God seems to be passive and silent concerning the suffering of believers caused by the enemies of God. However, God's sovereignty transcends all secular and supernatural powers. The power of God manifests itself in the acts of Jesus the Messiah whose power is paradoxically proclaimed by his being described as both a lion and a lamb.

\section{Einleitung}

In ihrer umfangreichen Monographie ,Die Namen des Vaters' analysiert Christiane Zimmermann entscheidende Gottesbezeichnungen des Neuen Testaments. In diesem Zusammenhang bietet die Autorin auch ein Kapitel zum Titel ,Pantokrator', zu seiner Verwendung in paganen und frühjüdischen Kontexten und seiner Bedeutung im Neuen Testament. Die entscheidenden Ergebnisse der Studie Zimmermanns lassen sich folgendermaßen zusammenfassen (Zimmermann 2007):

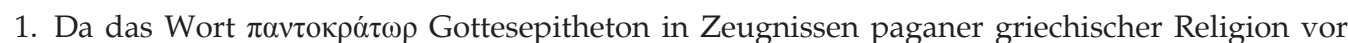
dem Aufkommen des Christentums nicht belegt ist, scheint eine Ableitung des christlichen Gebrauchs aus diesem Hintergrund unwahrscheinlich (pp. 234-238). ${ }^{1}$

2. Im Sprachgebrauch der LXX wie auch anderem frühjüdischen Schrifttum griechischer Sprache

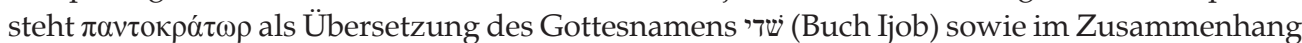
mit אúpı und $\theta \varepsilon o ́ \varsigma$ zur Übersetzung von צבאות (vgl. Zimmermann 2007:240-250). Interessant ist dabei besonders die Beobachtung, dass die LXX in ihrer Übersetzung der Tendenz folgte, gegenüber dem semantisch offeneren Text ihrer hebräischen Vorlage die göttliche Macht zu betonen:

... ein Vorgang, der vor dem Hintergrund des universalistischen Machtanspruchs der hellenistischen

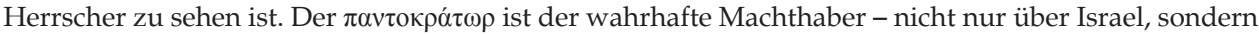

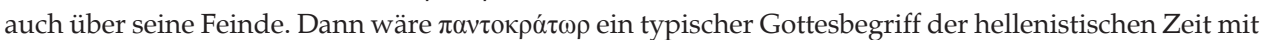
einer deutlich politischen Konnotation. (p. 247)

3. Die eben genannten politische Konnotation des Begriffs verstärkt sich noch einmal, wenn

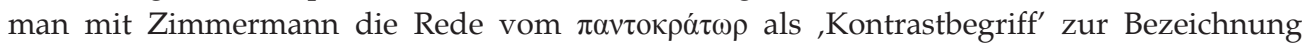
des römischen Kaisers als av̉ unternommen wird, ,eine bewusste Übersteigerung ... zu erzielen' (p. 240).

4. Die Betonung göttlicher Allmacht, mit Hilfe derer jegliche menschliche Macht relativiert wird,


als Gottesbezeichnung auftaucht. Dabei ist natürlich - neben einer Verwendung in 2 Korinther 6:18 - in allererster Linie an die Offenbarung des Johannes zu denken. In den insgesamt neun Vorkommen des Buchs der Offenbarung wiederum begegnet sieben Mal die vor allem im

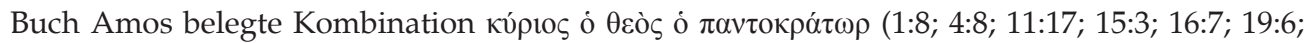

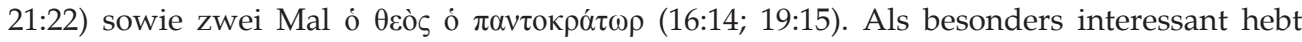
Zimmermann die erstmals in Offenbarung 1:8 begegnende Kombination der beiden dreiteiligen

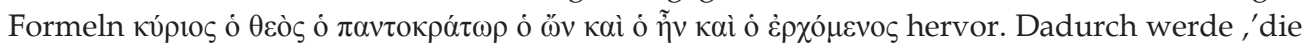
zeitliche Komponente der Macht Gottes und hier wiederum die Gegenwart Gottes besonders betont' (Zimmermann 2007:263). Daneben sei für die Apokalypse natürlich an die politische Dimension der Verwendung des Titels, mit dessen Hilfe der Text sich von den Ansprüchen des römischen Kaisers absetze, zu denken: ,Gott erweist sich allen irdischen Machthabern zum Trotz als der alleinige, über Zeit und Raum erhabene Herrscher der Welt, der schließlich seine himmlische Herrschaft aufrichten wird, in der herrschaftliche Strukturen letztlich nicht mehr nötig sind' (p. 268). 
Mit Zimmermanns Analyse der Verwendung des Titels ,Pantokrator' ist sicherlich bereits Entscheidendes zum Thema ,Gottes Macht' in der Apokalypse gesagt. Mit der Konzentration auf Hoheitstitel aber ist das Bild Gottes, das die Apokalypse entwirft, jedoch nur in Teilen beschrieben. Will man hier einen Schritt weiter kommen, empfiehlt sich neben der Analyse von Hoheitstiteln ein narrativer Zugang, der die Frage, welche Rolle Gottes Macht für den Text spielt, ins Gesamtgefüge der ,Erzählung' Apokalypse einordnet.

Meine These, die ich im Folgenden ausarbeiten werde, sieht dabei folgendermaßen aus:

Die Offenbarung des Johannes lässt sich als Antwort auf die in verschärfter Weise gestellte Theodizeefrage verstehen: ${ }^{2}$ Warum zeigt Gott ganz offensichtlich seine Macht nicht in der Weise, wie diejenigen, die unter der Macht der Welt zu leiden haben, dies von ihm erhoffen würden? Vor diesem Hintergrund entwickelt die Offenbarung des Johannes das Bild eines allmächtigen Gottes, des Pantokrators, der - obwohl er nicht zu handeln scheint - Handlungssouverän im kosmischen Drama bleibt und dieses zu einem guten Ende führt.

\section{Die Anfrage an Gott}

Bei aller gegenwärtigen Diskussion um den konkreten zeitgeschichtlichen Hintergrund der Entstehung der Offenbarung des Johannes besteht doch weitgehender Konsens darin, dass die von der Apokalypse angesprochenen Gemeinden sich als gesellschaftlich marginalisiert, ja bedrängt und verfolgt fühlen. ${ }^{3}$ Eine entscheidende Dimension dieser Situation scheint in dem Anspruch bestanden zu haben, der der Gemeinde in der zunehmenden Bedeutung des Kaiserkultes im Osten des Römischen Reiches entgegenschlug. ${ }^{4}$ Die große Frage, die die Gemeinden offensichtlich auch innerlich zu zerreißen drohte, bestand wohl in dem bis in das Alltagsleben hinein reichenden Problem, ob und wie weit man sich als Christ auf die religiös-politischen Ansprüche des römischen Imperiums, seiner Vertreter und der von ihnen kreierten Gesellschaft(en) einlassen dürfe, wo Kompromisse erlaubt seien und wo nicht. ${ }^{5}$ Bekanntlich steht die Seite des Sehers der Apokalypse für eine radikale, kompromisslose Haltung, eine Haltung, die zumindest in einem bekannten Fall - dem des Christen Antipas (Offb 2:13) - (und womöglich einer Anzahl nicht überlieferter Fälle) zum Martyrium geführt hat. ${ }^{6}$

Gerade diese Situation aber führt zur Formulierung einer scharfen Anfrage an Gott, die zwar nicht die Existenz 2.Im Grundansatz kommt meine Deutung der Apokalypse daher derjenigen nahe, die Wengst (2010) voraussetzt.

3.Diskutiert wird allerdings weiterhin, inwiefern sich die Gemeinden einer wirklichen Verfolgung ausgesetzt sehen und wie weit diese ging.

4.Zur Bedeutung des Kaiserkults für das Verständnis der Johannesapokalypse vgl. z.B. Frey (2006) und Giesen (2000). Zur Entwicklung des Kaiserkults in der Provinz Asia vgl. die entsprechende Studie von Witulski (2007).

5.Hierzu weiterführend u.a. Aune (2006).

6.Nicht klar wird, ob der Fall des ansonsten unbekannten Antipas tatsächlich den einzigen Fall eines Martyriums darstellt, den die angesprochenen Gemeinden erlitten, oder ob dieser Fall stellvertretend für andere genannt wird. DieBedrohung
scheint zumindest als so schlimm empfunden worden zu sein, dass sie Visionen wie scheint zumindest als so schlimm empfunden worden zu sein, dass sie Visionen wi Offenbarung 6:9-11 oder Offenbarung 20:4 rechtfertigt.
Gottes in Frage stellt, so doch die Weise seines Handelns (oder Nicht-Handelns) angeht. Das Problem besteht nicht nur darin, dass Menschen leiden (und zu Tode kommen), obwohl sie gerecht sind, sondern darin, dass Menschen zu Tode kommen, weil sie radikal an der Weisung Gottes festhalten und für ihn zu Zeugen werden. Ich verstehe Offenbarung 6:9-11 als eine Schlüsselszene für das gesamte Gottesverständnis der Offenbarung des Johannes: bei der Öffnung des fünften Siegels erblickt der Seher unter dem himmlischen Opferaltar die ,Seelen' derer, die ,wegen des Wortes Gottes und wegen des Zeugnisses, das sie gegeben hatten' (Offb 6:9), ,hingeschlachtet' wurden. ${ }^{7}$ In ihren Mund legt der Text einen Schrei, der nicht anders verstanden werden kann denn als Anfrage an das Handeln Gottes in einer Welt, deren Ungerechtigkeit so weit geht, dass in ihr gerade diejenigen zu Tode kommen, die auf Gottes Seite

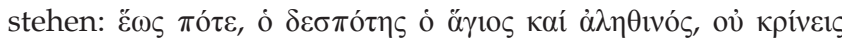

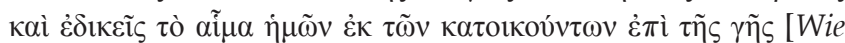
lange, oh heiliger und wahrer Herrscher, hältst du nicht Gericht und rächst nicht unser Blut aus denen, die auf der Erde wohnen?] (Offb 6:10).

Es lohnt sich, die konkrete Formulierung der Frage anzusehen. Anders als manche theologische Ansätze unserer Zeit stellt diese Form des Schreiens zu Gott nicht grundsätzlich dessen Göttlichkeit, beziehungsweise seine Allmacht in Frage. Vielmehr wird Gott sicherlich bewusst weiterhin als ,heiliger und wahrer Herrscher' angesprochen. Weiterhin vorausgesetzt wird, dass dieser Gott derjenige ist, der sich in seinem Gericht (über die gesamte Welt) letztlich als der gerechte Souverän erweisen wird. Angefragt wird also sicherlich nicht grundsätzlich die Allmacht Gottes, angefragt wird wohl auch nicht grundsätzlich seine Gerechtigkeit. Im Fokus steht vielmehr das Problem, dass sich diese Gerechtigkeit - wie wohl auch seine Macht - an denen, die auf seiner Seite stehen und gerade deswegen ,hingeschlachtet' werden, noch nicht erweist. Damit öffnet sich zwar nicht die Frage, ob Gott als allmächtig verstanden werden kann oder nicht, sondern das Problem, wie in einer Welt, in der Menschen für das Wort Gottes zu Tode kommen, weiterhin von Gottes Macht gesprochen werden kann.

Doch damit nicht genug: Offenbarung 6:9-10 spricht zwar vom Aufschrei der Hingeschlachteten zu Gott, vom Thron her aber scheint keine Antwort zu kommen - oder kann der Hinweis, dass sie nur noch, eine kurze Zeit' zu warten hätten, als etwas anderes als eine Vertröstung auffassen? ${ }^{8}$ Damit aber steht diese Frage über dem Teil der Apokalypse, der mit der Vision des himmlischen Thronsaals eingeleitet wird, und der letztlich in die Vision des himmlischen Jerusalem einmündet.

Die Bedeutung von Offenbarung 6:9-11 lässt sich aber noch genauer beschreiben: So versteht etwa Stefan Alkier $7 . Z u$ einem präziseren Verständnis der in dieser Szene erkennbaren Vorstellung einer Existenz nach dem Tode vgl. Nicklas (2009b).

8.Ich würde selbst so weit gehen, die Übergabe der weißen Gewänder noch nicht als wirklich befriedigende Antwort auf die Anfrage zu verstehen. Deutlich positiver formuliert allerdings Prigent (2004:275): [T] he gift of the robe should be understood as a first response to the prayer of the complete accomplishment of the divine plan of salvation; they receive as of now a deposit on the kingdom. This is possible ever since the eschatological work of Christ, and particularly his victory.' 
den Plot der Apokalypse als von der Überwindung eines 'Disäquilibriums' bestimmt:' ${ }^{9}$ Offenbarung 1:4-3:22 beschreibe eine Mangelsituation - d.h. die Situation, in der sich die Gemeinden Asias befinden - , die Kapitel 4-20 verstünden sich dann als Beschreibung von Handlungen, die diese Mangelsituation überwinden. Diese wiederum münden schließlich mit Kapitel 21-22 in die Beschreibung einer neuen Situation, die Darstellung eines ewigen Lebens, das nicht mehr von den in Kapiteln 1-3 beschriebenen Mängeln bestimmt sei. Auch wenn diese Überlegungen nicht jedes Detail im Text der Apokalypse zu erklären vermögen (und dies auch nicht anzielen), ist damit auf im Grunde geniale Weise eine Grundstruktur des 'Plot' der Apokalypse gezeichnet. Vielleicht aber könnte man noch einen Schritt weitergehen: der Mangel, von dem die Apokalypse ausgeht, besteht zumindest aus der im Text beschriebenen Sicht weniger in der äußeren politischen wie gesellschaftlichen Situation, in der die Gemeinden von Asia sich befinden. Diese bildet zwar einen Teil des Problems, dieses wird vom Text selbst aber bis zu seinem Urgrund, einem nicht angemessenen Verhältnis großer Teile der Menschheit zu Gott und einem Harren der bedrängten Gemeinden auf die Parusie und die damit verbundene Durchsetzung der Gerechtigkeit Gottes, zurückgeführt. Das Zueinander dieser Gedanken findet sich im Grunde schon in Offenbarung 1:9 angesprochen, wo sich Johannes als ,Mitgenosse' der Angesprochenen'in Bedrängnis und Ausharren auf Jesus' versteht, der sich wegen seines Christuszeugnisses auf der Insel Patmos befindet. Aus der im Text beschriebenen himmlischen Perspektive werden die Gemeinden in Kapitel 2 und 3 ermahnt, durchzuhalten und sich ihrer Gottes- wie Christusbeziehung gemäß zu verhalten. Die andere Perspektive - nämlich die der Gemeinden, die zu Gott schreien, dass er gegen die bedrohenden Mächte der Welt nun seine Gerechtigkeit erweisen soll - kommt, soweit ich sehe, am besten in Offenbarung 6:9-11 zum Tragen. Zumindest ein entscheidender Aspekt des im Text der Offenbarung zu überwindenden Disäquilibriums besteht also in der Erfahrung, dass die Gemeinden zwar aufgrund ihrer ,Werke' beurteilt werden (vgl. 2:2, 19; 3:1, 8, 15), dass Gott selbst aber in einer Welt, in der den Gemeinden die verschiedensten Machtansprüche begegnen, nicht (oder nicht in der erwarteten Weise) zu handeln scheint.

\section{Der Gott, der nicht zu handeln scheint}

Tatsächlich scheint der Gott der Apokalypse seltsam entzogen und - betrachtet man die direkt mit Gott in Verbindung gebrachten Aktionen - überaus passiv. In dem in Kapitel 4 beschriebenen himmlischen Thronsaal konzentriert, ja definiert sich alles von seinem Verhältnis zum Thron Gottes. Eine Gestalt Gottes selbst, von dem es heißt, dass er , wie ein Jaspis und Karneol aussah,' (Offb 4:3) aber wird nicht greifbar, er ist nicht einmal direkt als ,Gott', sondern alleine als derjenige, der , auf dem Thron sitzt' (Offb 4:3, 9), bezeichnet. ${ }^{10}$ 9.Vgl. Knapp in Alkier (2010:280-281), sowie ausführlicher in Alkier (2011:289-294).

10.Damit zeigt der Text auch eine Weiterentwicklung gegenüber seinem hier entscheidenden Intertext, der Thronsaalvision des Ezechiel. Zur Ezechielrezeption der Apokalypse weiterführend vgl. Hieke (2004).
Dies ist besonders auffällig, da alle anderen in der Szene beschriebenen Figuren sich in permanenter - man könnte sagen ,ewiger' - Aktion befinden. So heißt es von den vier Lebewesen am Thron Gottes, dass sie ,niemals ruhen' (Offb 4:8b), während gleichzeitig die vierundzwanzig Ältesten in dauernder Proskynese und Anbetung verharren und dabei ihre goldenen Kränze vor dem Thron Gottes niederlegen. Der (scheinbaren) Passivität dessen, der auf dem Thron sitzt und letztlich unzugänglich erscheint, kontrastiert somit die ewige Aktivität aller anderen Beteiligten. Doch bereits die Thronsaalszene selbst durchbricht dieses Bild. Besonders interessant scheint mir das Zueinander zweier Attribute, die zwei Mal kurz hintereinander wiederholt werden: sowohl Offenbarung 4:9, als auch 4:10 sprechen von Gott einerseits als dem (1) auf dem Thron Sitzenden, als auch (2) dem in alle Ewigkeit Lebenden, dem nicht nur die vier Lebewesen am Thron, sondern auch die vierundzwanzig Ältesten vor dem Thron die Ehre erweisen. Meine These ist, dass diese beiden Gottesattribute zwei Aspekten der Darstellung Gottes in der Apokalypse entsprechen, die miteinander verschränkt sind: dem Aspekt des absolut souverän Herrschenden, dessen Handeln dem Menschen entzogen zu sein scheint, und dem Aspekt des lebendigen und in alle Ewigkeit handelnden Gottes. Vielleicht muss in diesem Zusammenhang zudem auch an die Doxologie der vierundzwanzig Ältesten in Offenbarung 4:11 erinnert werden. Dort ist davon die Rede, dass Gott derjenige sei, der ,das All' ( $\tau \grave{\alpha} \pi \alpha \dot{v} v \tau \alpha)$ erschaffen habe und dies ,durch seinen Willen' geschehen sei. Die Idee der Schöpfung aufgrund des göttlichen Willens ist bekanntlich in frühjüdischer Literatur kaum belegt (vgl. allerdings Dan 4:35, 37 Theodotion-Fassung $)^{11}$, sie fügt sich hier aber gerade deswegen besonders gut in den Kontext, weil sie die Idee des Handelns Gottes mit der Tatsache verbindet, dass dieses Handeln nicht im Sinne eines Handwerkens zu verstehen ist. Der Gott der Apokalypse ist kein Demiurg, wie man ihn vielleicht aufgrund von Aussagen aus dem zweiten Schöpfungsbericht der Genesis missverstehen könnte. Er ist vielmehr der souverän, beziehungsweise als Souverän Handelnde.

\section{Das Handeln Gottes durch das Lamm}

Auch Kapitel 5 spielt im himmlischen Thronsaal. In die, soweit dies literarisch möglich ist, ,Ewigkeit' darstellende Szene gerät Bewegung. In der Krise um die Buchrolle, die niemand öffnen kann (Offb 5:3) wird nun offensichtlich ein mehr oder minder ,punktuelles', einmaliges Ereignis beschrieben, das in eine Szene mündet, die man vielleicht am besten als Einsetzung des Lammes in eine Machtstellung, ja vielleicht sogar ,Inthronisation', verstehen kann.

Auch das Lamm wird durch zwei Gruppen von Attributen beschrieben, die - zumindest im weitesten Sinne - als ,aktiv', und ,passiv' einzuordnen sind: Einerseits ist es bezeichnet als ,Löwe aus dem Stamm Juda' (Offb 5:5; vgl. Gen 49:9),



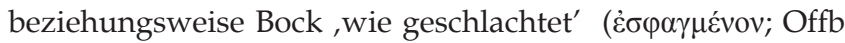
11.Zur Bedeutung von Schöpfungsaussagen als Grundmotiv der Johannesoffenbarung vgl. Nicklas (2010a). 
5:6). Aus der Hand dessen, von dem es nun wieder heißt, dass er , auf dem Thron saß' (Offb 5:7), empfängt es das geheimnisvolle Buch, dessen Siegel es nun öffnen wird. ${ }^{12}$ Dass damit derjenige, der ', in alle Ewigkeit lebt' (Offb $4: 9,10)$ das ,Lamm, das geschlachtet wurde', in eine Position von ,Macht einsetzt beziehungsweise, besser ihm Macht überträgt (ohne dabei selbst Macht abzugeben), zeigen die nun folgenden Doxologien verschiedener Gruppen in konzentrischen Kreisen um den Thron: Offenbarung 5:9-11 preist die vergangenen Leistungen des Lammes, Offenbarung 5:12 spricht von seinem Machtempfang und Offenbarung 5:13 stellt es in Parallele zu dem, der auf dem Thron sitzt, Offenbarung 5:14 schließlich spricht von einer Proskynese der 24 Ältesten. Vor dem Hintergrund des eben Gesagten scheint kaum denkbar, dass diese nun nicht auch dem Lamm gilt, auch wenn der Text hier offen formuliert ist. ${ }^{13}$ Interessant ist, dass Gott im fünften Kapitel nur noch als der auf dem Thron Sitzende beschrieben ist. Sicherlich bedeutet das Wegfallen des zweiten Attributs - der in alle Ewigkeit Lebende - nicht, dass dies nun nicht mehr gilt, vielleicht aber kann daran gedacht werden, dass Gott derjenige ist, der sein machtvolles Handeln als Lebendiger nicht nur am Lamm erwiesen hat, sondern (von nun an) im Handeln des Lammes zeigt.

Das Handeln des Lammes besteht nun einerseits im Öffnen der Siegel des Buches aus Gottes Hand, welches in die beiden weiteren Visionszyklen um die Posaunen und Schalen mündet. In dieser Verkettung von Ereignissen, die in den Visionen geschaut werden und die verschiedene Aspekte des Kosmos betreffen, mit dem Handeln Christi, der die Siegel des Buches öffnet, welches er aus der Hand dessen empfangen hat, der , auf dem Thron sitzt', wird im Grunde gezeigt, dass der nicht zu handeln scheinende Gott ist, von dem alles Handeln ausgeht.

Andererseits findet sich das Lamm auch in den Visionenzyklen selbst als Handelnder. Auch wenn sich nicht eindeutig belegen lässt, wer der Reiter auf dem weißen Pferd ist, der nach dem Brechen des ersten Siegels (Offb 6:2) als Sieger ausreitet, um zu siegen, so macht es doch meines Erachtens den besten Sinn, ihn als Christus selbst zu verstehen, ${ }^{14}$ der am Ende des mit Offenbarung 6 einsetzenden Spannungsbogens bei der Parusie erneut als Reiter einzieht, um in der letzten Schlacht (Offb 19:11-21) die Gottesfeinde zu besiegen und so das tausendjährige Messiasreich (Offb 20:46) zu ermöglichen. Möchte der Text damit zum Ausdruck bringen, dass der Christus, der als Lamm im himmlischen Thronsaal geschaut und in Macht und Herrlichkeit verehrt wurde, verborgen als Sieger weiterhin in der Geschichte der Menschheit handelt, um zu siegen? Dies würde sehr gut zu dem Bild bei der Berufung des Sehers der Apokalypse passen, als er Christus inmitten der sieben Leuchter, die für 12.Interessanterweise spricht Giesen (1997:159), von der Buchrolle als dem, Symbol der Macht', die an Christus übergeben wird.

13.Giesen (1997:172) schreibt: ,So kehrt der Lobpreis zurück zur ewigen Anbetung Gottes im Himmel (4:8-9), die nun den erhöhten Christus einschließt.'

14.Eine solche Deutung der Szene vertritt etwa Herzer (1999). die Gemeinden stehen, erblickt (Offb 1:13). Damit in Bezug stehen wohl auch die in Offenbarung 7:9-17 und 14:1-5 in die Siebenerzyklen eingebauten Visionen, in denen das zukünftige Heil derer, die dem Lamm ,nachfolgen', schon vor der Vision des himmlischen Jerusalem geschaut und mit ihrer jetzigen Situation in Bezug gesetzt wird. ${ }^{15}$

Siegen' wiederum heißt für die Apokalypse nicht, den Kampf gegen die Gottesfeinde mit den gleichen Mitteln wie diese aufzunehmen und sie zu bezwingen - ihre Vorstellung des ,Sieges' ist vielmehr eine gebrochene. ${ }^{16}$ Sie orientiert sich am Vorbild des Lammes, das wie geschlachtet ist und sich gerade so als der siegreiche Löwe von Juda erweist. Dieser Gedanke hält sich durch bis hin zur Beschreibung der Schlacht in Offenbarung 19:11-21(siehe Nicklas 2009a), die zwar als ,blutige Angelegenheit' in dem Sinne dargestellt ist, dass Christus, der erneut als Reiter auf einem weißen Pferd beschrieben ist, ein blutiges Gewand trägt (Offb 19:13), ein Motiv, das aber gerade dann im Kontext der Apokalypse am meisten Sinn macht, wenn es sich um das Blut Christi selbst handelt. Mit ihm erringen diejenigen, die ihm nachfolgen und die nun weiße Gewänder tragen (Offb 19:14) - kaum nur Engelheere, sondern diejenigen, die Christus durch ihr Zeugnis gefolgt sind - den entscheidenden Sieg in einer Schlacht, von der kein einziger Schwertstreich geschildert wird, sondern von der nur ihr Ergebnis festgehalten wird (Offb 19:17-21). Mehrere Motive im Text machen deutlich, dass dieser Sieg Christi und derer, die ihm folgen, der Sieg Gottes ist: so kann der Gedanke, dass der Reiter einen Namen hat, den allein er trägt, nur als Hinweis auf den unaussprechbaren Gottesnamen verstanden werden; Offenbarung 19:13 wiederum bezeichnet ihn als ,Wort Gottes', 19:15 spricht vom Zorn Gottes, der sich in seinem Tun an den Feinden erweist, Offenbarung 19:17 deutet den Sieg in der Schlacht als ,Mahl Gottes'. Die in Offenbarung 5-6 angedeutete Kette zwischen dem Geschehen auf der Erde, dem Handeln Christi und seinem Ursprung in Gott zeigt sich also auch hier.

\section{Die Inszenierung der ,Macht' der Gottesfeinde}

In das Gesamtbild dieser Inszenierung der Macht Gottes, der - obwohl er nicht zu handeln scheint - als der eigentliche Handlungssouverän der bis zu Neuschöpfung und himmlischem Jerusalem, also bis zum ,happy end' führenden Ereignisse vorgestellt ist, fügt sich auch die vor allem in den Kapiteln 12 und 13 begegnende Inszenierung der Gottesfeinde ein. Der Text spricht zunächst von gewaltigen Zeichen am Himmel, etwa dem beängstigenden Drachen, der die Himmelsfrau, die für das Gottesvolk steht, zu verschlingen droht. So bedrohlich die Szenerie wirkt (und damit sicherlich den beängstigenden Szenerien korrespondiert, denen sich die Gemeinden ausgesetzt

15.Diese Interpretation ist allerdings umstritten - vertreten wird auch die Ansicht, dass die beiden Visionen bereits den jetzigen Zustand der christlichen Gemeinden zum Ausdruck bringen wollen. Dagegen aber spricht meines Erachtens schon die zum Ausdruck bringen wollen. Dagegen aber sprich
Lokalisation von Offenbarung 14:1-5 auf dem Zion.

16.Zur Rede vom ,Sieg' in der Offenbarung des Johannes vergleiche weiterführend Taeger (2006). 
fühlen), so gebrochen ist auch die hier verwendete Bildwelt. Geschildert wird nicht ein Götterkampf mit mehr oder weniger offenem Ende. Vor dem Hintergrund von Intertexten der LXX wird vielmehr klar, dass der Drachen ein zwar für den Menschen bedrohliches Geschöpf ist, aber eben doch nur Geschöpf, dessen zerstörerische Macht die Schöpfung Gottes nicht wirklich gefährden kann. ${ }^{17}$ Dieses Bild aber wird noch einmal gebrochen: die Tatsache, dass der Drache, der für ,die alte Schlange, die Teufel oder Satan heißt' (Offb 12:9), steht, so sehr ,wütet' (vgl. Offb 12:12), ist nicht Zeichen seines Sieges, sondern Folge seiner endgültigen Niederlage, die er - erneut nicht im Götterkampf, sondern im Kampf gegen die Engel Gottes - erlitten hat, eine Niederlage, die gleichwohl

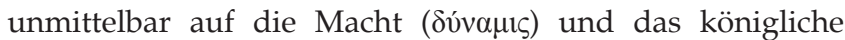
Herrschen ( $\beta \alpha \sigma i \lambda \varepsilon i ́ \alpha)$ Gottes wie auch die Vollmacht ( $\dot{\xi} \xi o v \sigma i ́ \alpha)$ rückgeführt wird (Offb 12:10).

Und trotzdem ist die Apokalypse nicht so unrealistisch, dem Drachen keine Macht zuzusprechen - die Machtentfaltung des römischen Imperiums (und seiner ,totalitären' Anforderungen an den Menschen) steht den Adressaten des Textes ja offensichtlich dauernd vor Augen. Kapitel 13 erzählt, wie der Drache seine Macht an Helfershelfer, das Tier aus dem Meer wie auch das Tier aus der Erde, verleiht. So heißt es vom ersten Tier, dass der Drache ihm ,seine



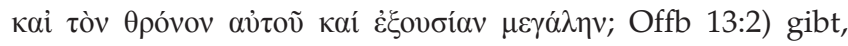
während das zweite Tier, die gesamte Vollmacht des ersten Tieres ausübt' ( 13:12). Abgründig allerdings ist die Reihe von vier passiven

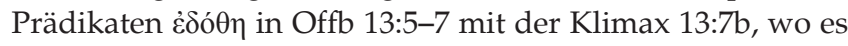
heißt, dass dem ersten Tier ,Vollmacht über jeden Stamm, jedes Volk, jede Zunge und jede Nation gegeben wurde.' Wer ist als Subjekt dieser Passive (vgl. auch 13:15) zu verstehen? Im Kontext könnte man zunächst an den Drachen denken, von dem ja auch die Macht des Tieres ausgeht. Doch warum formuliert der Text dann in solch offener Weise? Muss nicht vielmehr an ein Passivum Divinum gedacht werden? Dann geht auch die Macht des Tieres letztlich auf Gott zurück, von dem alle Macht ausgeht. Die Formulierung liegt dann auf einer Linie mit der Aussage des johanneischen Jesus im Dialog mit Pilatus (Joh 19:11): ,Du hättest keine Macht über mich, wenn es dir nicht von oben gegeben worden wäre ...' Das Tier bliebe damit teuflisches, dämonisches Tier - und trotzdem würde seine Macht in geheimnisvoller Weise auf den einen Gott zurückgeführt. ${ }^{18}$

So gefahrvoll das in Kapitel 13 geschilderte Szenario auch wirkt, so sehr relativiert sich das bedrohliche Potenzial des Textes vor dem Hintergrund seiner alttestamentlichen Intertexte, vor allem Daniel 7 (sehe Hieke 2012), und wird, im Zusammenhang mit Offenbarung 4 gelesen,

17.Zur Verwendung des Wortes $\delta$ pók $\omega v$ in der LXX vergleiche Eynikel (2004). Zu einer Gesamtinterpretation der Szene vergleiche Nicklas (2006) - mit weiterführender Literatur.

18.Zur Deutung vergleiche auch Satake (2008:299), der schreibt: ,Der Geber' dieser Befugnisse ist trotz Vers 2 nicht der Drache, sondern Gott ... Der Verfasser will den Lesern deutlich machen, dass auch das Wirken des Tieres, das Gott zu verspotten scheint, unter strenger Kontrolle Gottes steht.' Wichtig hierzu auch die Gedanken von Wengst (2010:137), der deutlich macht, dass wir es hier nicht mit einem ,apokalyptischen Dualismus' zu tun haben, sondern der Text weiterhin auf dem Boden biblischen Schöpfungsglaubens steht. geradezu zur Karikatur pseudo-göttlichen Herrschens. Die Machtausübung des Drachens und der mit ihm verbundenen Tiere wird so als Teil im Plan Gottes entlarvt, der zu Endgericht und Heil für diejenigen führen wird, die ,Standhaftigkeit und Glaubenstreue' (Offb 13:10) zeigen. Sicherlich nicht zufällig begegnet hier erneut das Motiv der

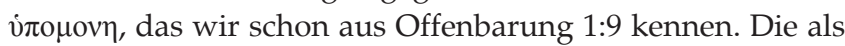
Offenbarung autorisierte Karikatur - man könnte geradezu von einer ,Entmythologisierung' sprechen - der sich selbst mythifizierenden Macht des Staats wird so zum Baustein eines Gesamtbildes, für das die Idee der Macht Gottes zentral ist, das aber gleichzeitig jegliche irgendwie , anthropomorph' missverständlichen Bilder eines in seinen Handlungen selbst Hand anlegenden Gottes vermeidet.

\section{Das Sprechen Gottes}

Zumindest indirekt lassen sich die Visionen der Kapitel 4-19 so als Antwort auf den Schrei aus Offenbarung 6:9-11 verstehen; diese Antwort aber gibt noch nicht (oder zumindest nicht direkt oder in der erhofften Weise) Gott selbst, der weiterhin als schweigend beschrieben ist. Der Gott der Apokalypse reagiert auf das Schreien der Dahingeschlachteten in Offenbarung 6:9-11 in anderer, weniger direkter Weise, als dies etwa auf die Klagen Ijobs hin erzählt wird. Das Schweigen des Gottes der Apokalypse hält sich durch und ist bei allen Hinweisen auf sein die Welt vorandrängendes, Partei ergreifendes Handeln auszuhalten: die Apokalypse ordnet die von ihren Lesern erfahrenen Katastrophen in den Plan des Handlungssouveräns Gottes ein - doch bleiben sie in der Erfahrung der Leser Katastrophen. So ist das Buch der Apokalypse sicherlich Hoffnungsbuch, aber keineswegs naiv.

Innerhalb des Textes wird das Schweigen dessen, zu dem die Dahingeschlachteten schreien, erst in Offenbarung 21:5 gebrochen: ,Siehe, ich mache alles neu!' Die Antwort von Seiten Gottes, der schweigt und deswegen passiv scheint, ist diejenige, dass er seine Schöpfung zu einem guten Ende führt. Dies ist in engem Zusammenhang zu Offenbarung 21:3-4 zu sehen, wo Prophezeiungen aus dem Alten Testament neu aufgenommen werden (vor allem Ezechiel 37:27 und Jesaja 25:8):

Seht, die Wohnung Gottes unter den Menschen! Er wird in ihrer Mitte wohnen und sie werden sein Volk sein; und er, Gott, wird bei ihnen sein. Er wird alle Tränen von ihren Augen abwischen: Der Tod wird nicht mehr sein, keine Trauer, keine Klage, keine Mühsal. (Offb 21:3-4)

Auch wenn es jetzt nicht so $\mathrm{zu}$ sein scheint, steht der machtvolle Gott Israels $\mathrm{zu}$ seinen Verheißungen. Angesprochen ist dabei genau der Gedanke, der zum Beginn der Apokalypse als Disäquilibrium gekennzeichnet war - die gebrochene Beziehung des Menschen zu Gott, die zu Tränen, Trauer, Klage und schließlich Tod führt, wird durch das unmittelbare Wohnen Gottes - und des Lammes - unter den Seinen (vgl. auch Offb 21:22; 22:1) auf ewig in Ordnung gebracht.

Damit aber ist noch nicht alles gesagt: auch wenn die ,Erzählung' der Apokalypse einen schweigenden, entzogenen 
Gott zu beschreiben scheint - und damit wohl Erfahrungen ihrer Leserinnen und Leser aufgreift - versteht sie sich selbst in zwar gebrochener, vermittelter Weise auf Gott zurückgehend (sehe Nicklas 2011) und macht dies bereits in ihren ersten Worten klar: ,Offenbarung Jesu Christi, die Gott ihm gegeben hat, ...' (Offb 1:1). Das Bild des jetzt zu schweigen scheinenden, in Wirklichkeit aber souverän handelnden und am Ende der Zeiten sein endgültiges schöpferisches ,MachtWort' sprechenden Gottes wird auf Gott selbst zurückgeführt. Mit anderen Worten: die Apokalypse führt sich selbst auf das durch Christus vermittelte Sprechen dieses Gottes zurück. ${ }^{19}$

Damit aber nimmt sie selbst auf machtvolle Weise Stellung in einem Machtkonflikt: die Frage nach dem angemessenen Verhalten im Gegenüber zu den Ansprüchen von Welt und Gesellschaft scheint in den Gemeinden Asias auf unterschiedliche Weise beantwortet worden zu sein. Der radikalen ,Partei' des Sehers entgegen standen Gruppen, deren eigene Stimme heute nur noch sehr gebrochen und verzerrt in einigen Aussagen der Sendschreiben zu rekonstruieren ist und unter denen offenbar eine Prophetin, die Johannes mit der alttestamentlichen Chiffre Isebel belegt (Offb 2:20; vgl. 1 Könige 16), eine Rolle spielte (vgl. Aune 2006). Mit seiner in der Apokalypse erfolgenden Inszenierung von Gottes Macht, die er auf Gottes Wort selbst zurücknimmt, spricht der Seher selbst ein prophetisches ,Macht-Wort', das von seiner Seite her das letzte Wort im Konflikt der Gemeinden zu sein beansprucht.

\section{Fazit}

Die Proklamation des Gottes der Apokalypse als Pantokrator ordnet sich nicht nur in den Gemeinden der Apokalypse von außen bedrängenden Konflikt ein: sicherlich wird mit der Darstellung Gottes als ,Allherrscher' jeglicher quasigöttlicher Anspruch des römischen Imperiums (wie auch aller anderer totalitären Mächte) auf den Menschen entlarvt und aufs Schärfste zurückgewiesen. So ist die Apokalypse als theologisches Werk mit einem Schwerpunkt auf einer Theologie der Schöpfung, die geradezu organisch mit Eschatologie verbunden ist, auch politischer Text.

Über diese sicherlich richtigen und zutreffenden Gedanken hinaus kann die Darstellung des Gottes der Apokalypse auch an sich von höchster theologischer Bedeutung über die konkrete Situation ihrer Entstehung hinaus verstanden werden. Der Text lässt sich als Auseinandersetzung mit der in Offenbarung 6:9-11 explizit gestellten Frage nach dem Handeln Gottes in Macht und Gerechtigkeit verstehen. Der Text macht sich die Antwort darauf keineswegs leicht: er beschreibt vielmehr einen gegenüber allem menschlichen Schreien scheinbar schweigenden, ja passiv erscheinenden Gott, deutet dies aber nicht als Zeichen von Gottes Ohnmacht, sondern verknüpft es mit der Vorstellung, dass der lebendige Gott in absolut souveräner Weise seine Macht durchsetzt. Diese aber verwirklicht sich nicht in einer Form, die einfach Abbild der Machtausübung der Gottesfeinde ist, sondern im Handeln Christi, der gerade als Lamm, das geschlachtet 19.Zum damit verbundenen Autoritätsanspruch der Offenbarung des Johannes vergleiche Nicklas (2010b). wurde, siegreicher Löwe von Juda ist. An diesem Sieg haben letztlich auch diejenigen teil, die dem Lamm auf seinem Weg folgen und so selbst an seiner Herrschaft im Messiasreich Anteil erhalten. Dieses Ziel wird auch da erreicht, wo den Kräften des Bösen scheinbare Übermacht gewährt scheint. Selbst diese wird im Letzten auf Gott, den Schöpfer, selbst zurückgeführt, bleibt jedoch auch dann teuflische, sich wider Gott stellende Macht. Gerade mit ihren dunklen Bildern bietet die Apokalypse so keineswegs billige Vertröstung, sie nimmt vielmehr genau die Schrecken wahr, denen die Angesprochenen ausgesetzt sind und konfrontiert den schweigenden Gott damit. Trotzdem kann sie als Hoffnungsbuch verstanden werden, das seine Hoffnung auf die Macht des schöpferischen Pantokrators setzt. Gleichwohl führt es seine Hoffnungsbilder nicht naiv an den Katastrophen dieser Welt vorbei, sondern durch sie hindurch - und verkündet Gott als denjenigen, der in geheimnisvoller Weise durch Christus unter seinen bedrängten Gemeinden ist und für sie handelt.

Das Ringen um das Verständnis der Macht Gottes aber findet nicht im mehr oder minder luftleeren Raum statt: mit dem Anspruch, prophetisches Buch zu sein, das sich letztlich auf die über Christus vermittelte Initiative Gottes zurückführt, der durch das Buch in die Gemeinden hinein ,spricht', versucht die Apokalypse selbst, ,Macht' in einem Konflikt auszuüben, der die Christen Asias zu zerreißen droht. Indem sie die Gegner ganz auf die Seite des Bösen zieht, das sich gegen die Macht Gottes stellt, nimmt sie ihnen die Stimme. Mit welchem Recht dies geschehen sein mag, lässt sich heute historisch nicht mehr ergründen - mit ihrem Anspruch auf prophetische Macht aber hat die Apokalypse sich letztlich durchgesetzt. ${ }^{20}$

\section{Konkurierende interessen}

Der Verfasser erklärt hiermit, daß er keinerlei finanzielle oder persönliche Bindung hat, die ihn zweckwidrig beim Schreiben dieses Artikels beeinflusste.

\section{Bibliographie}

Alkier, S., 2010, Neues Testament, Uni Taschenbücher (UTB) Basics, Francke, Tübingen.

Alkier, S., 2011, 'The Book of Re(ve)lation: How to read intertextually', in J. Verheyden, T. Nicklas \& A. Merkt (eds.), Interpreting violent texts: Ancient Christian commentators of the Book of Revelation, pp. 287-303, NOTA 92, Vandenhoeck commentators of the Book
\& Ruprecht, Göttingen.

Aune, D.E., 2006, 'The social matrix in the Apocalypse of John', in D.E. Aune (ed.), Apocalypticism, prophecy and magic in Early Christianity: Collected essays, pp. 175-189, WUNT 199, Mohr, Tübingen.

Eynikel, E., 2004, 'The use of "dragon" in the Septuagint', in B. Taylor, J.A.L. Lee, P.R. Burton \& R.E. Whitaker (eds.), Biblical Greek language and lexicography: Essays in honor of Frederick W. Danker, pp. 126-135, Eerdmans, Grand Rapids, MI.

Frey, J., 2006, 'The relevance of the Roman Imperial cult for the Book of Revelation: Exegetical and hermeneutical reflections on the relation between the seven letters and the visionary part of the book', in J. Fotopoulos (ed.), The New Testament and Early Christian literature in Greco-Roman context: Studies in honor of David E. Aune, pp. 231-255, NovT.S 122, Brill, Leiden.

Giesen, H., 1997, Die Offenbarung des Johannes, RNT, Pustet, Regensburg.

Giesen, H., 2000, 'Das Römische Reich im Spiegel der Johannes-Apokalypse', in H. Giesen (Hrsg.), Studien zur Johannesapokalypse, pp. 100-213, SBAB 29, Katholisches Bibelwerk, Stuttgart.

20.Ich bin einigen Kollegen, vor allem Wim Weren und Pieter de Villiers, für kritische Rückfragen zu meinem Beitrag zu Dank verpflichtet; ich habe versucht, die entsprechenden Gedanken einzubauen und so den Beitrag weiter zu entwickeln. 
Herzer, J., 1999, 'Der erste apokalyptische Reiter und der König der Könige: Ein Beitrag zur Christologie der Johannesapokalypse', New Testament Studies 45, 230-249. zur Christologie der Johannesapokalypse', New

Hieke, T., 2004, 'Der Seher Johannes als neuer Ezechiel: Die Offenbarung des Johannes vom Ezechielbuch her gelesen', in D. Sänger (Hrsg), Das Ezechielbuch in der Johannesoffenbarung, pp. 1-30, BThSt 76, Neukirchener Verlag, NeukirchenVluyn.

Hieke, T., 2012, 'The Reception of Daniel 7 in the Revelation of John', in R. Hays \& S. Alkier (ed.), The Book of Revelation: Theology, Politics, and Intertextuality, pp. 47-67, Baylor, Waco, TX.

Nicklas, T., 2006, 'Frau, Lamm und Drache in der Offenbarung des Johannes' Sacra Scripta 4, 43-72.

Nicklas, T., 2009a, 'Der Krieg und die Apokalypse: Gedanken zu Offb 19:11-21, 150165 ', in A. Holzem (Hrsg.), Krieg und Christentum: Religiöse Gewalttheorien in der Kriegserfahrung des Westens, n.p., KriG 50, Schöningh, Paderborn.

Nicklas, T., 2009b, “"Die Seelen der Geschlachteten” (Offb 6,9)? Zum Problem leibliche Auferstehung in der Offenbarung des Johannes', in T. Nicklas, F.V. Reiterer \& J. Verheyden (Hrs.). The human body in death and resurrection, pp. 329-349, DCLY, De Gruyter, Berlin.

Nicklas, T., 2010a, 'Schöpfung und Vollendung in der Offenbarung des Johannes', in T. Nicklas \& K. Zamfir (eds.), Theologies of creation in Early Judaism and Ancient Christianity: In honour of Hans Klein, pp. 389-414, DCL Studies 6, De Gruyter, Berlin.
Nicklas, T., 2010b, "The Words of the Prophecy of this Book": Playing with Scriptural Authority in the Book of Revelation', in M. Popovic (ed.), Authoritative Scriptures in Ancient Judaism, pp. 309-326, JSJ.S 141, Brill, Leiden.

Nicklas, T., 2011, 'Der Ewige spricht in die Zeit: Gotteswort und Menschenwort in der Offenbarung des Johannes, Sacra Scripta 9 [im Druck].

Prigent, P., 2004, Commentary on the Apocalypse of St. John, Mohr, Tübingen.

Satake, A., 2008, Die Offenbarung des Johannes, KeK, Vandenhoeck \& Ruprecht, Göttingen.

Taeger, J-W., 2006, “'Gesiegt! O himmlische Musik des Wortes! Zur Entfaltung des Siegesmotivs in den johanneischen Schriften', in J-W., Taeger (Hrsg.), Johanneische Perspektiven. Aufsätze zur Johannesapokalypse und zum johanneischen Kreis 1984-2003, pp. 81-104, FRLANT 215, Vandenhoeck \& Ruprecht, Göttingen.

Wengst, K., 2010, “Wie lange noch?" Schreien nach Recht und Gerechtigkeit - eine Deutung der Apokalypse des Johannes, Kohlhammer, Stuttgart.

Witulski, T., 2007, Kaiserkult in Kleinasien: Die Entwicklung der kultisch-religiösen Kaiserverehrung in der römischen Provinz Asia von Augustus bis Antoninus Pius, NTOA 63, Vandenhoeck \& Ruprecht, Göttingen.

Zimmermann, C., 2007, Die Namen des Vaters: Studien zu ausgewählten neutestamentlichen Gottesbezeichnungen vor ihrem frühjüdischen und paganen Sprachhorizont, AJEC 69, Brill, Leiden. 